\title{
On the Square Integrability of Quasi Regular Representation on Semidirect Product Groups
}

\author{
A.A. Arefijamaal · R.A. Kamyabi-Gol
}

Received: 15 April 2008 / Published online: 14 March 2009

(C) Mathematica Josephina, Inc. 2009

\begin{abstract}
Let $H$ be a locally compact group and $K$ be a locally compact abelian group. Also let $G=H \times{ }_{\tau} K$ denote the semidirect product group of $H$ and $K$, respectively. Then the unitary representation $\left(U, L^{2}(K)\right)$ on $G$ defined by $U(h, x) f(y)=$ $\delta(h)^{\frac{1}{2}} f\left(\tau_{h^{-1}}\left(y x^{-1}\right)\right)$ is called the quasi regular representation. The properties of this representation in the case $K=\left(\mathbb{R}^{n},+\right)$, have been studied by many authors under some specific assumptions. In this paper we aim to consider a general case and extend some of these properties when $K$ is an arbitrary locally compact abelian group. In particular we wish to show that the two conditions (i) $\delta \Delta_{H} \not \equiv 1$, and (ii) the stabilizers $H^{\omega}$ are compact for a.e. $\omega \in \widehat{K}$; both are necessary for square integrability of $U$. Furthermore, we shall consider some sufficient conditions for the square integrability of $U$. Also, for the square integrability of subrepresentations of $U$, we will introduce a concrete form of the Duflo-Moore operator.
\end{abstract}

Keywords Semidirect product · Fourier transform · Locally compact abelian (LCA) group $\cdot$ Square integrable representation

Mathematics Subject Classification (2000) Primary 43A65 · Secondary 42C40

\section{Preliminaries and Introduction}

Throughout this article $G$ will denote a locally compact group. It is very well known that such a group possesses a left Haar measure $\mu_{G}$, that is unique up to a mul-

\footnotetext{
A.A. Arefijamaal (凶)

Department of Mathematics, Tarbiat Moallem University, Sabzevar 397, Iran

e-mail: arefijamal@sttu.ac.ir

R.A. Kamyabi-Gol

Department of Mathematics, Faculty of Science, Islamic Azad University-Mashhad Branch, Mashhad, Iran

e-mail: kamyabi@ferdowsi.um.ac.ir
} 
tiplication by constants. By $L^{p}(G), 1 \leq p<\infty$ will denote the Banach space of all equivalent classes of a.e. defined complex valued $\mu_{G}$ measurable functions $f$ on $G$ such that $\|f\|_{p}=\left(\int_{G}|f(x)|^{p} d \mu_{G}(x)\right)^{1 / p}<\infty$. For any locally compact group $G$, there exists a continuous homomorphism $\Delta_{G}: G \longrightarrow(0,+\infty)$ such that $\int_{G} f(x y) d \mu_{G}(x)=\Delta_{G}\left(y^{-1}\right) \int_{G} f(x) d \mu_{G}(x)$, for all $f \in L^{1}(G) ; \Delta_{G}$ is called the modular function of $G, G$ is called unimodular if $\Delta_{G} \equiv 1$.

A unitary representation of $G$ is a homomorphism $\pi: G \longrightarrow \mathcal{U}(\mathcal{H})$ where $\mathcal{H}$ is a non-zero Hilbert space and $\mathcal{U}(\mathcal{H})$ is the group of all unitary operators on $H_{\pi}$ which is continuous with respect to the strong operator topology. If $M$ is a non-zero closed and $\pi$-invariant subspace of $\mathcal{H}$, the restriction of $\pi$ to $M, \pi_{M}(x):=\left.\pi(x)\right|_{M}$ defines a representation of $G$ on $M$, called a subrepresentation of $\pi$. One of the well known continuous unitary representation on a locally compact group is the left regular representation which is defined as $L: G \longrightarrow \mathcal{U}\left(L^{2}(G)\right), x \longmapsto L_{x}$ where $L_{x} f(y)=f\left(x^{-1} y\right)$ for all $x, y \in G$ and all $f \in L^{2}(G)$. A unitary representation $\pi$ on a Hilbert space $\mathcal{H}$ is called irreducible if the only closed subspaces of $\mathcal{H}$ that are invariant under $\pi(x)$, for all $x \in G$ are $\{0\}$ and $\mathcal{H}$. Usually the left regular representation is not irreducible, for more details one can refer to [6]. The irreducible unitary representations of a locally compact group $G$ are the basic building blocks of the harmonic analysis associated to $G$. If $\pi$ is an irreducible unitary representation of $G$ on a Hilbert space $\mathcal{H}$, we wish to find a vector $\psi$ in $\mathcal{H}$ such that

$$
\int_{G}\langle\eta, \pi(x) \psi\rangle \pi(x) \psi d \mu_{G}(x)=\eta, \quad \forall \eta \in \mathcal{H} .
$$

Such a vector $\psi$ is called an admissible vector. The existence of an admissible vector is not generally guaranteed [13]. However, (1) is equivalent to $\int_{G}|\langle\psi, \pi(x) \psi\rangle|^{2} d \mu_{G}(x)<\infty$ or $\int_{G}|\langle\eta, \pi(x) \psi\rangle|^{2} d \mu_{G}(x)<\infty$ for all $\eta \in \mathcal{H}$ (for more details see [2]). The irreducible unitary representation $\pi$ is called square integrable if at least one nonzero admissible vector in $\mathcal{H}$ exists. It can be shown that if $\pi$ is square integrable then there exists a dense set of admissible vectors in $\mathcal{H}$. Moreover, if $G$ is unimodular then every nonzero vector is admissible and consequently (1) holds for all $\psi$ in $\mathcal{H}$.

Now fix an admissible vector $\psi$ in $\mathcal{H}$. If we define $W_{\psi}: \mathcal{H} \longrightarrow L^{2}(G)$ by

$$
\left(W_{\psi} \eta\right)(x)=C_{\psi}^{\frac{-1}{2}}\langle\eta, \pi(x) \psi\rangle, \quad \eta \in \mathcal{H}, x \in G,
$$

where $C_{\psi}=\frac{1}{\|\psi\|^{2}} \int_{G}|\langle\psi, \pi(x) \psi\rangle|^{2} d \mu_{G}(x)$, then $W_{\psi}$ has the following properties [2]:

- $W_{\psi}$ is a linear isometry onto a closed subspace $\mathcal{H}_{\psi}$ of $L^{2}(G)$.

- $W_{\psi}$ intertwines $\pi$ and the left regular representation $L$, (i.e. $W_{\psi} \pi(x)=L_{x} W_{\psi}$, $\forall x \in G)$.

- The adjoint of $W_{\psi}$ and $W_{\psi}^{-1}$ are coincide on $\mathcal{H}_{\psi}$.

- The inversion formula holds i.e.

$$
\int_{G}\left(W_{\psi} \eta\right)(x) \pi(x) \psi d \mu_{G}(x)=C_{\psi} \eta, \quad \forall \eta \in \mathcal{H} .
$$

$W_{\psi}$ is known as the continuous wavelet transform. 
For example when $G$ is the Affine group, which is the semidirect product of the two locally compact abelian (LCA) groups $(\mathbb{R} \backslash\{0\},$.$) and (\mathbb{R},+)$ with the group operations

$$
(a, b)(c, d)=(a c, b+a d), \quad(a, b)^{-1}=\left(\frac{1}{a}, \frac{-b}{a}\right)
$$

the quasi regular representation $U$ of $G$ on the Hilbert space $L^{2}(\mathbb{R})$ defined by $(U(a, b) f)(x)=a^{\frac{-1}{2}} f\left(\frac{x-b}{a}\right)$, is an irreducible unitary representation of $G$. A vector $\psi \in L^{2}(\mathbb{R})$ is an admissible vector for $U$ if $C_{\psi}^{2}=\int_{\mathbb{R}} \frac{|\widehat{\psi}(\omega)|^{2}}{|\omega|} d \omega<\infty$, where $\widehat{\psi}$ denotes the Plancherel transform of $\psi$ [13]. Hence according to [5] for an admissible vector $\psi$ we can define the continuous wavelet transform $W_{\psi}$ by $W_{\psi} f(a, b)=$ $\langle f, U(a, b) \psi\rangle$ where $f \in L^{2}(\mathbb{R}), a \in \mathbb{R} \backslash\{0\}, b \in \mathbb{R}$ and rewrite the inversion formula (2) as follows:

$$
\int_{\mathbb{R} \backslash\{0\}} \int_{\mathbb{R}}\langle f, U(a, b) \psi\rangle U(a, b) \psi \frac{d a d b}{a^{2}}=C_{\psi} f .
$$

The wavelet transform has rich theoretical structures and is extremely useful as tools for building signal transforms, adapted to various signal geometries, quantum mechanics, ... [2, 7]. Continuous wavelet transform admits a quite natural generalization to locally compact groups. Such a unified approach seems to be useful since it emphasizes on a clear way the basic features of continuous wavelet transform and includes all cases important for applications. Although a lot of the basic results also hold for locally compact groups, the general theory for these groups is considerably more complicated.

It should be noted that generally the quasi regular representation $U$ is not irreducible. For example, in the Affine group where $G=(0,+\infty) \times{ }_{\tau} \mathbb{R}, U$ is not irreducible. But one can still find a class of square integrable subrepresentations of the quasi regular representation (see Theorem 2.9 of [3]). H. Fuhr in [8] and S.T. Ali et al. in [1] have considered the semidirect product $H \times{ }_{\tau} \mathbb{R}^{n}$ when $H$ is a closed subgroup (but not necessarily abelian) of $G L(n, \mathbb{R})$. They proved that compactness of some special kinds of stabilizers are necessary for the square integrability of subrepresentations of $U$. Moreover, they have shown in this case the Duflo-Moore operator can be rewritten as a multiple of modular function of the group $H \times{ }_{\tau} \mathbb{R}^{n}$. In this article we extend these results for the group $H \times{ }_{\tau} K$ where $H$ and $K$ are locally compact groups, and $K$ is also abelian.

\section{Main Results}

For two locally compact groups $H$ and $K$ where $K$ is also abelian, let $h \longmapsto \tau_{h}$ be a homomorphism of $H$ into the group of automorphisms of $K \operatorname{denoted}$ by $\operatorname{Aut}(K)$. Also assume that the mapping $(h, x) \longmapsto \tau_{h}(x)$ from $H \times K$ onto $K$ is continuous. Then the set $H \times K$ endowed with the product topology and the operations:

$$
(h, x) .\left(h^{\prime}, x^{\prime}\right)=\left(h h^{\prime}, x \cdot \tau_{h}\left(x^{\prime}\right)\right),
$$




$$
(h, x)^{-1}=\left(h^{-1}, \tau_{h^{-1}}\left(x^{-1}\right)\right)
$$

is a locally compact group. This group is called the semidirect product of $H$ and $K$, respectively, and is denoted by $H \times{ }_{\tau} K$. Let $G=H \times_{\tau} K$. Then the left Haar measure of $G$ is $d \mu_{G}(h, x)=\delta(h) d \mu_{H}(h) d \mu_{K}(x)$ and $\Delta_{G}(h, x)=\delta(h) \Delta_{H}(h)$ is its modular function, in which $\delta$ is a positive continuous homomorphism on $H$ and is given by

$$
\mu_{K}(E)=\delta(h) \mu_{K}\left(\tau_{h}(E)\right),
$$

for all measurable subsets $E$ of $K$. Next we define the quasi regular representation on a semidirect product group.

Definition 2.1 According to the above notations, the quasi regular representation $\left(U, L^{2}(K)\right)$ on $G=H \times_{\tau} K$ is defined by $U(h, x) f(y)=\delta(h)^{\frac{1}{2}} f\left(\tau_{h^{-1}}\left(y x^{-1}\right)\right)$ where $f \in L^{2}(K),(h, x) \in G$ and $y \in K$.

$U$ is not generally irreducible [13]. Consider $\widehat{K}$ as the dual group of the LCA group $K$ and denote its left Haar measure by $d \omega$. Then one can define a continuous action from $H$ on $\widehat{K}$ by $(h, \omega) \mapsto \omega \circ \tau_{h}$. Now for a fix $\omega \in \widehat{K}$, the stabilizer and the orbit of $\omega$, that play a key role in our discussion are defined by

$$
H^{\omega}:=\left\{h \in H ; \omega \circ \tau_{h}=\omega\right\}, \quad O_{\omega}:=\left\{\omega \circ \tau_{h} ; h \in H\right\},
$$

respectively. $H^{\omega}$ is a closed subgroup of $H$ and $O_{\omega}$ is an $H$-invariant subset in $\widehat{K}$.

A measurable invariant subset $A$ of $\widehat{K}$ with positive measure is called ergodic if the only measurable invariant subset of $A$ with positive measure is $A$. For any measurable invariant subset $A$ of $\widehat{K}$ with positive measure define

$$
L_{A}^{2}(K):=\left\{f \in L^{2}(K) ; \operatorname{supp}(\widehat{f}) \subseteq A\right\} .
$$

It is a closed $U$-invariant subspace of $L^{2}(K)$ and therefore the restriction of $U$ to $L_{A}^{2}(K)$, denoted by $U_{A}$, is a subrepresentation of $U$. Moreover, if $A$ is ergodic then $U_{A}$ is irreducible. In [3], it is shown that every irreducible subrepresentation of $U$ is equivalent to a representation of this form. Therefore the quasi regular representation $U$ is irreducible if there is any nontrivial invariant subset in $\widehat{K}$. We are interested in finding an equivalent condition depending on the structure of orbits. To do so, first we need a couple of lemmas:

Lemma 2.2 Consider the semidirect product $H \times_{\tau} K$. Then for every $g \in L^{1}(K) \cap$ $L^{2}(K), h \in H$ and $\omega \in \widehat{K}$ we have;

$$
\begin{gathered}
\left.\left(g \circ \tau_{h}\right) \hat{(} \omega\right)=\delta(h) \widehat{g}\left(\omega \circ \tau_{h^{-1}}\right), \\
d\left(\omega \circ \tau_{h^{-1}}\right)=\delta(h) d \omega .
\end{gathered}
$$

Proof Let $g \in L^{1}(K)$ then there exists a sequence $\left\{g_{n}\right\}$ in $C_{c}(K)$, the space of all continuous and compact supported functions on $K$, such that $g_{n} \longrightarrow g$ in the norm 
topology of $L^{1}(K)$. It is clear that $g_{n} \circ \tau_{h} \in C_{c}(K)$ for all $h \in H$ and $n \in \mathbb{N}$. Moreover, (3) implies that

$$
\left\|g_{n} \circ \tau_{h}-g \circ \tau_{h}\right\|_{1}=\delta(h)\left\|g_{n}-g\right\|_{1} .
$$

That is, $g \circ \tau_{h} \in L^{1}(K)$. Now a straightforward calculation gives (4). To obtain (5), note that $d\left(\omega \circ \tau_{h}\right)$ is a translation invariant measure on $\widehat{K}$ and if $g \in L^{1}(K) \cap L^{2}(K)$, then

$$
\begin{aligned}
\int_{\widehat{K}}|\widehat{g}(\omega)|^{2} d \omega & =\int_{K}|g(x)|^{2} d \mu_{K}(x)=\delta\left(h^{-1}\right) \int_{K}\left|g\left(\tau_{h}(x)\right)\right|^{2} d \mu_{K}(x) \\
& =\delta\left(h^{-1}\right) \int_{\widehat{K}} \mid\left(\left.g \circ \tau_{h} \widehat{)}(\omega)\right|^{2} d \omega=\delta(h) \int_{\widehat{K}}\left|\widehat{g}\left(\omega \circ \tau_{h^{-1}}\right)\right|^{2} d \omega .\right.
\end{aligned}
$$

Lemma 2.3 Let $H$ be a locally compact group and $f \in L^{1}(H)$ be non-negative. If $\int_{C} f(x) d \mu_{H}(x)=0$, for every compact subset $C$ of $H$, then $f \equiv 0$.

The proof is straightforward.

Definition 2.4 A nonzero $\psi$ in $L^{2}(K)$ is called a wavelet vector for the quasi regular representation $\left(U, L^{2}(K)\right)$ if $C_{\psi}^{2}:=\int_{H}\left|\widehat{\psi}\left(\omega \circ \tau_{h}\right)\right|^{2} d h<\infty$ is constant for a.e. $\omega \in \widehat{K}$.

It is worthwhile to note that every wavelet vector is an admissible vector [3].

For an LCA group $K$ the mapping $T(x)=x^{2}$ is a continuous homomorphism from $K$ onto $K^{2}:=T(K)$, a subgroup of $K$. This map is not open, in general. For example, let $K_{1}=\prod_{i=1}^{\infty} \mathbb{Z}_{2}$ be endowed with the product topology. Then consider $K=\prod_{i=1}^{\infty} \mathbb{Z}_{4}$ and regard $K_{1}$ as a subgroup of $K$, where we identify the elements of $\mathbb{Z}_{2}$ with the elements of $\mathbb{Z}_{4}$ of order $\leq 2$. We endow $K$ with the topology such that $K_{1}$ is an open and compact subgroup of $K$. Obviously $\left(K_{1}\right)^{2}=\{0\}$ and $K^{2}=K_{1}$. Hence $T$ is not open.

Nevertheless, a reasonably large class of continuous homomorphisms are automatically open, as Theorem 5.29 of [11] shows. For instance, if $K$ is a $\sigma$-compact locally compact abelian group such that $K^{2}$ is closed, then $T$ is open. Recall that, every open subgroup is automatically closed. Also it is worthwhile to note that for every LCA group $K$ with open component of identity, $K^{2}$ is open, (combine Theorems 24.25 and 24.30 of [11]). In particular, $K^{2}$ is open if $K$ is an LCA Lie group.

The next proposition will be needed in the sequel.

Proposition 2.5 Let $K$ be an LCA group such that the mapping $T: K \longrightarrow K^{2}$; $x \longmapsto x^{2}$ is a topological isomorphism. Then there exists a constant $C_{K}>0$ such that $\mu_{K}(E)=C_{K} \mu_{K}\left(E^{2}\right)$ for any measurable subset $E$ of $K$. In this case, if $M$ is an open subgroup of $K$ then $C_{M}=C_{K}$.

Proof First note that $K^{2}$ is an open subgroup of $K$. Hence its Haar measure is the restriction of $\mu_{K}$ to $K^{2}$. On the other hand, if we define the set function $v$ on $K^{2}$ by $v(E)=\mu_{K}\left(T^{-1}(E)\right)$ for any Borel set $E$ of $K^{2}$, then $v$ is a Borel Radon measure 
which is also translation invariant, that is, $v(a E)=v(E)$ for all $a \in K^{2}$. Hence by the uniqueness of the Haar measure, there is a constant $C_{K}>0$ such that $\nu=C_{K} \mu_{K}$. This completes the proof of the first part. For the second part, consider a non-empty compact subset $A$ of $M$ then we have

$$
C_{M}=\frac{\mu_{K}(A)}{\mu_{K}\left(A^{2}\right)}=C_{K}
$$

Corollary 2.6 With assumptions in the Proposition 2.5

(i) If $K$ is discrete then $C_{K}=1$.

(ii) If $K$ has a compact subgroup with positive measure (in particular, $K$ is compact) then $C_{K} \geq 1$.

For the remainder, we assume that $T: K \longrightarrow K^{2} ; x \longmapsto x^{2}$, is a topological isomorphism, namely $K$ is topologically isomorphic to its open subgroup $K^{2}$.

It is worthwhile to recall that there exists a topological isomorphism $T_{0}$ from $\mathbb{R}^{n} \times$ $L$ onto $K$ where $L$ is an LCA group containing a compact open subgroup $L_{0}$ and the unique nonnegative integer $n$ is the covering dimension of $K$ as a topological space (see Theorem 24.30 of [11]). Let $\mu_{\mathbb{R}^{n}}$ and $\mu_{L}$ be the Haar measures of $\mathbb{R}^{n}$ and $L$, respectively. Then by Proposition 2.5 there exists a constant $C_{K}>0$ such that

$$
\left(\mu_{\mathbb{R}^{n}} \times \mu_{L}\right)(E)=C_{K} \mu_{K}\left(T_{0} E\right),
$$

for all measurable subsets $E$ of $\mathbb{R}^{n} \times L$. Put $E=\prod_{i=1}^{n}\left[a_{i}, b_{i}\right] \times L_{0}$, then

$$
\begin{aligned}
C_{K} \mu_{K}\left(\left(T_{0} E\right)^{2}\right) & =C_{K} \mu_{K}\left(T_{0} E^{2}\right)=\left(\mu_{\mathbb{R}^{n}} \times \mu_{L}\right)\left(E^{2}\right) \\
& =\mu_{\mathbb{R}^{n}}\left(\prod_{i=1}^{n}\left[2 a_{i}, 2 b_{i}\right]\right) \cdot \mu_{L}\left(L_{0}^{2}\right)=2^{n} \frac{1}{C_{L_{0}}} C_{K} \mu_{K}\left(T_{0} E\right) .
\end{aligned}
$$

Therefore $C_{K}=\left(\frac{1}{2}\right)^{n} C_{L_{0}}$. Now assume that $\omega \longmapsto \omega^{2}$ is a topological isomorphism from $\widehat{K}$ onto $(\widehat{K})^{2}$, then similarly $C_{\widehat{K}}$ is also a multiple of two numbers; one of them less than or equal to 1 and another one $\geq 1$. For example, if $K$ is topologically isomorphic with $\mathbb{R}^{n} \times L_{0}$, in which $L_{0}$ is a compact group, then Corollary 2.6 implies that $C_{\widehat{K}}=\frac{1}{2^{n}}$. It is easy to see that if $C_{K}$ and $C_{\widehat{K}}$ exist, then $C_{K}=C_{\widehat{K}}$. Moreover $C_{K}=C_{\widehat{K}}=1$ iff $K$ is discrete or has a compact open subgroup.

In [14], R. S. Laugesen et al. have introduced necessary and sufficient conditions to generate wavelet vectors associated to the quasi regular representation of $H \times{ }_{\tau} \mathbb{R}^{n}$. The next theorem generalize this result.

Theorem 2.7 Let $G=H \times{ }_{\tau} K$ and $\left(U, L^{2}(K)\right)$ has a wavelet vector $\psi$. Then

(i) The stabilizer of $\omega$ is compact for a.e. $\omega \in \widehat{K}$.

(ii) $\delta \Delta_{H} \not \equiv 1$, if $C_{\widehat{K}} \neq 1$. In particular, $G$ is non-unimodular.

(iii) For every $\omega \in \widehat{K}$ that its orbit $A:=O_{\omega}$ has positive measure, the subrepresentation $U_{A}$ is square integrable. 
Proof Let $\psi$ be a wavelet vector. Then $C_{\psi}^{2}=\int_{H}\left|\widehat{\psi}\left(\omega \circ \tau_{h}\right)\right|^{2} d h<\infty$, for a.e. $\omega \in \widehat{K}$. By contrary, assume that $H^{\omega}$ is not compact for some $\omega \in \widehat{K}$. Let $C \subset H$ be compact and $h_{1} \in H^{\omega}$. Then $H^{\omega} \nsubseteq h_{1} C C^{-1}$, so there exists $h_{2} \in H^{\omega}$ such that $h_{2} \notin h_{1} C C^{-1}$. By induction we obtain a collection of mutually disjoint compact sets $\left\{h_{n} C\right\}$ with $h_{n} \in H^{\omega} \backslash \bigcup_{j=1}^{n-1} h_{j} C C^{-1}, n \geq 2$. Then

$\infty>C_{\psi}^{2}=\int_{H}\left|\widehat{\psi}\left(\omega \circ \tau_{h}\right)\right|^{2} d h \geq \sum_{n} \int_{h_{n} C}\left|\widehat{\psi}\left(\omega \circ \tau_{h}\right)\right|^{2} d h=\sum_{n} \int_{C}\left|\widehat{\psi}\left(\omega \circ \tau_{h}\right)\right|^{2} d h$.

Hence $\int_{C}\left|\widehat{\psi}\left(\omega \circ \tau_{h}\right)\right|^{2} d h=0$ for all compact subsets $C$ of $H$. Now Lemma 2.3 implies $\widehat{\psi}=0$ which is a contradiction. To prove (ii) assume that $\delta \Delta_{H}=1$. Fix a wavelet vector $\psi \in L^{2}(K)$; since the constant $C_{\psi}$ is independent of $\omega \in \widehat{K}$ we have

$$
\begin{aligned}
C_{\psi}^{2}\|\psi\|_{2}^{2} & =\int_{H}\left|\widehat{\psi}\left(\omega^{2} \circ \tau_{h}\right)\right|^{2} d \mu_{H}(h) \int_{\widehat{K}}|\widehat{\psi}(\omega)|^{2} d \omega \\
& =\int_{\widehat{K}} \int_{H}\left|\widehat{\psi}\left(\omega^{2} \circ \tau_{h}\right)\right|^{2}|\widehat{\psi}(\omega)|^{2} d \mu_{H}(h) d \omega \\
& =\int_{\widehat{K}} \int_{H}\left|\widehat{\psi}\left(\omega^{2}\right)\right|^{2}\left|\widehat{\psi}\left(\omega \circ \tau_{h^{-1}}\right)\right|^{2} \delta(h) d \mu_{H}(h) d \omega \\
& =\int_{\widehat{K}}\left|\widehat{\psi}\left(\omega^{2}\right)\right|^{2} \int_{H}\left|\widehat{\psi}\left(\omega \circ \tau_{h}\right)\right|^{2} \delta\left(h^{-1}\right) \Delta\left(h^{-1}\right) d \mu_{H}(h) d \omega \\
& =\int_{\widehat{K}}\left|\widehat{\psi}\left(\omega^{2}\right)\right|^{2} d \omega . C_{\psi}^{2} \\
& =C_{\psi}^{2}\|\psi\|_{2}^{2} C_{\widehat{k}} .
\end{aligned}
$$

Which it is impossible. Finally (iii) is a consequence of (i) and Theorem 2.10 of [3].

Note that the necessary conditions (i) and (ii) in the Theorem 2.7 are not sufficient. In fact, let $H=\left\{2^{j} a: j \in \mathbb{Z}, a \in S L(2, \mathbb{Z})\right\}$. Then $\left(U, L^{2}\left(\mathbb{R}^{2}\right)\right)$ on $H \times_{\tau} \mathbb{R}^{2}$ doesn't have any admissible vector. Moreover, $\Delta_{H} \delta \not \equiv 1$ and for a.e. $\omega \in \widehat{\mathbb{R}^{2}}$ the stabilizer is compact [14]. R.S. Laugesen et al. introduced the sufficient conditions when $K=\mathbb{R}^{n}$ (see Theorem 4.1). It also holds when $K$ is compactly generated abelian Lie group.

Now we are confronted with a question: When is the quasi regular representation square integrable? To answer this question, we require some information about orbits. If $K$ is an LCA group then:

- $O_{1_{\widehat{K}}}=\left\{1_{\widehat{K}}\right\}$.

- For any $\omega \in \widehat{K}, O_{\omega}$ is an $H$-invariant subset of $\widehat{K}$.

- $O_{\omega}=O_{\gamma}$ iff $O_{\omega} \cap O_{\gamma} \neq \phi$.

- There exists $\omega \in \widehat{K}$ such that $O_{\omega}=\widehat{K} \backslash\left\{1_{\widehat{K}}\right\}$ iff $O_{\omega}=\widehat{K} \backslash\left\{1_{\widehat{K}}\right\}$ for all $\omega \neq 1_{\widehat{K}}$.

Proposition 2.8 Let $U$ be the quasi regular representation associated to the semi direct product group $G=H \times_{\tau} K$ and $\widehat{K} \backslash\left\{1_{\widehat{K}}\right\}=O_{\omega}$ for some $\omega \in \widehat{K}$. Then $U$ is irreducible. Moreover, if there exists an open orbit, then $O_{\gamma}=\widehat{K}$ a.e. for some $\gamma \in \widehat{K}$. 
Proof If $O_{\omega}=\widehat{K} \backslash\left\{1_{\widehat{K}}\right\}$ then $U$ is irreducible since there is no nontrivial invariant subset in $\widehat{K}$ (see [3]). If $U$ is irreducible and the orbit of $\gamma$ has positive measure for some $\gamma$ in $\widehat{K}$, then $A:=O_{\gamma}$ is an ergodic subset in $\widehat{K}$ and $L_{A}^{2}(K)$ will be an $U$ invariant closed subspace of $L^{2}(K)$. Now irreducibility of $U$ implies that $L_{A}^{2}(K)=$ $L^{2}(K)$, hence $O_{\gamma}=A=\widehat{K}$ a.e.

Corollary 2.9 If there exists $\gamma \in \widehat{K}$ with open orbit then the quasi regular representation $U$ is square integrable iff $O_{\gamma}=\widehat{K} \backslash\left\{1_{\widehat{K}}\right\}$ and the stabilizer of $\omega$ is compact for a.e. $\omega \in \widehat{K}$.

Notice that the affine group $\mathbb{R} \backslash\{0\} \times_{\tau} \mathbb{R}$ satisfies Corollary 2.9.

\section{Duflo-Moore Operator for Square Integrable Subrepresentations of Quasi Regular Representation}

Let $G$ be a locally compact group and $U$ a square integrable representation of $G$ on the Hilbert space $\mathcal{H}$. Then there exists a unique positive, self adjoint, invertible operator $C$ on $\mathcal{H}$ such that its domain is the set of all admissible vectors that is dense in $\mathcal{H}$. If $\psi_{1}$ and $\psi_{2}$ are two admissible vectors and $\phi_{1}$ and $\phi_{2}$ are arbitrary vectors in $\mathcal{H}$, then

$$
\int_{G} \overline{\left\langle U(g) \psi_{1}, \phi_{1}\right\rangle}\left\langle U(g) \psi_{2}, \phi_{2}\right\rangle d \mu_{G}(g)=\left\langle C \psi_{2}, C \psi_{1}\right\rangle\left\langle\phi_{1}, \phi_{2}\right\rangle .
$$

Furthermore, $G$ is unimodular iff $C$ is a multiple of the identity. The operator $C$ is called the Duflo-Moore operator and (6) is referred as the orthogonality relation [1, 10].

Now assume that $H$ is a locally compact group and $K$ is an LCA group. Let $G=H \times{ }_{\tau} K$ denote the semidirect product of $H$ and $K$ respectively, and let $\left(U, L^{2}(K)\right)$ be the quasi regular representation of $G$. Fix $\gamma \in \widehat{K}$ and take $A=O_{\gamma}$. In [3] it is shown that $A$ has positive measure and the stabilizers of $A$ are compact iff the subrepresentation $\left(U_{A}, L_{A}^{2}(K)\right)$ is square integrable. In this case every wavelet vector in the sense of Definition 2.4 will be an admissible vector. Our aim is to find a concrete form for Duflo-Moore operator associated to the square integrable subrepresentations of $U$. For the remainder of this section, we restrict our attention to the case that the natural map $h H^{\gamma} \longrightarrow \gamma \circ \tau_{h^{-1}}$ is a homeomorphism. In [9] Glimm has found some equivalent conditions to this property.

Lemma 3.1 If $\left(U_{A}, L_{A}^{2}(K)\right)$ is a square integrable subrepresentation of $U$ and $\psi \in$ $L_{A}^{2}(K)$ is an admissible vector, then

$$
C_{\psi}^{2}=c_{0} \int_{A}|\widehat{\psi}(\omega)|^{2} c(\omega) d \omega
$$

where $c(\omega)=\Delta(h) \delta(h)$ defines a continuous function on $A$ and $c_{0}$ is a constant. 
Proof The map $T_{\gamma}: H \longrightarrow O_{\gamma} ; h \mapsto \gamma \circ \tau_{h}$ transfer $\mu_{H}$ to a measure on $O_{\gamma}$. In fact $\nu(E)=\mu_{H}\left(T_{\gamma}^{-1}(E)\right)$ for measurable subsets $E$ of $O_{\gamma}$ defines a regular Borel measure $v$ on $O_{\gamma}$. Moreover a straightforward calculation gives $T_{\gamma}^{-1}\left(E \circ \tau_{h}\right)=T_{\gamma}^{-1}(E) h$ where $E \circ \tau_{h}=\left\{\omega \circ \tau_{h} ; \omega \in E\right\}$ is the image of Borel measurable subset $E$ of $O_{\gamma}$ under the natural action of $H$ on $\widehat{K}$. Hence, if $\nu_{h}$ is defined by $v_{h}(E)=v\left(E \circ \tau_{h^{-1}}\right)$ then the Radon-Nikodym derivative is given by

$$
\frac{d \nu_{h}}{d v}=\Delta\left(h^{-1}\right)
$$

For a moment let us denote by $\lambda$ the Haar measure of LCA group $\widehat{K}$. Then by (3) we have:

$$
\frac{d \lambda_{h}}{d \lambda}=\delta(h)
$$

On the other hand, since $H / H^{\gamma}$ and $O_{\gamma}$ are homeomorphic, one can consider the strongly quasi invariant measures $\widetilde{v}$ and $\widetilde{\lambda}$ on the homogeneous space $H / H^{\gamma}$ as the image of $v$ and $\lambda$, respectively. But by Theorem 2.59 of [6] every strongly quasi invariant measure arises from a rho-function on $H$. Therefore, there are two rho-functions $\rho_{\nu}$ and $\rho_{\lambda}$ such that

$$
\begin{aligned}
\int_{H} f\left(\gamma \circ \tau_{h^{-1}}\right) \rho_{\nu}(h) d \mu_{H}(h) & =\int_{H / H^{\gamma}} \int_{H^{\gamma}} f\left(\gamma \circ \tau_{a^{-1} h^{-1}}\right) d a d \widetilde{\nu}\left(h H^{\gamma}\right) \\
& =\int_{H^{\prime} H^{\gamma}} f\left(\gamma \circ \tau_{h^{-1}}\right) d \widetilde{\nu}\left(h H^{\gamma}\right)=\int_{O_{\gamma}} f(\omega) d \nu(\omega),
\end{aligned}
$$

in which $\mathrm{f}$ is measurable function on $O_{\gamma}$ and $d a$ is the normalized Haar measure on compact group $H^{\gamma}$. A similar expression holds for $\rho_{\lambda}$, hence by Theorem 2.56 of [6]

$$
\rho_{\nu}(h)=\rho_{\nu}\left(1_{H}\right) \Delta_{H}\left(h^{-1}\right), \quad \rho_{\lambda}(h)=\rho_{\lambda}\left(1_{H}\right) \delta(h) .
$$

Furthermore, the Radon-Nikodym derivative can be calculated as $\frac{d v}{d \lambda}\left(\gamma \circ \tau_{h}\right)=$ $\frac{\rho_{\lambda}\left(1_{H}\right)}{\rho_{\nu}\left(1_{H}\right)} \Delta(h) \delta(h)$. Finally take $c_{0}=\rho_{\lambda}\left(1_{H}\right)$ then we have:

$$
\begin{aligned}
C_{\psi}^{2}=\int_{H}\left|\widehat{\psi}\left(\gamma \circ \tau_{h}\right)\right|^{2} d \mu_{H}(h) & =\frac{1}{\rho_{\nu}\left(1_{H}\right)} \int_{O_{\gamma}}|\widehat{\psi}(\omega)|^{2} d \nu(\omega) \\
& =c_{0} \int_{A}|\widehat{\psi}(\omega)|^{2} \Delta(h) \delta(h) d \lambda(\omega) .
\end{aligned}
$$

Now by combining (6) and (7) we can obtain the Duflo-Moore operator as follows;

Theorem 3.2 If $A=O_{\gamma}$ is an open orbit for some $\gamma \in \widehat{K}$ and $C$ is the Duflo-Moore operator associated to square integrable subrepresentation $\left(U_{A}, L_{A}^{2}(K)\right)$ then there exits a $c_{0}>0$ such that

$$
\left(C \psi \hat{)}\left(\gamma \circ \tau_{h}\right)=\left(c_{0} \delta(h) \Delta(h)\right)^{\frac{1}{2}} \widehat{\psi}\left(\gamma \circ \tau_{h}\right),\right.
$$

for all $\psi$ in $L_{A}^{2}(K)$ and $h \in H$. 


\section{Examples}

In this section we review some examples of semidirect products. Let $H$ be a locally compact group and there exists a continuous homomorphism $\tau$ from $H$ to $G L(n, \mathbb{R})$. Many authors have been studied the quasi regular representation associated to the group $H \times{ }_{\tau} \mathbb{R}^{n}$, see $[1,4,7,12]$. In this case the best results are due to Laugesen and et al. [14].

Theorem 4.1 (R.S. Laugesen et al. [14]) Let $H$ be a $\sigma$-compact, locally compact group, and $h \longmapsto \tau_{h}$ from $H$ to $G L(n, \mathbb{R})$ be a continuous homomorphism. Take $G=$ $H \times{ }_{\tau} \mathbb{R}^{n}$, for $\omega \in \widehat{\mathbb{R}^{n}}$ and $\epsilon>0$ let

$$
H_{\epsilon}^{\omega}=\left\{h \in H ;\left\|\omega \circ \tau_{h}-\omega\right\| \leq \epsilon\right\}
$$

be the $\epsilon$-stabilizer of $\omega$. Then

(i) If the quasi regular representation of $G$ is square integrable, then $\Delta_{H} \not \equiv \delta^{-1}$ and $H_{0}^{\omega}$, the stabilizer of $\omega$, is compact for a.e. $\omega \in \mathbb{R}^{n}$.

(ii) If $\Delta_{H} \not \equiv \delta^{-1}$ and for a.e. $\omega \in \mathbb{R}^{n}$ there exists an $\epsilon>0$ such that the $\epsilon$-stabilizer of $\omega$ is compact, then the quasi regular representation of $G$ has an admissible vector.

By using Theorem 4.1, we can construct some examples of semidirect products $H \times{ }_{\tau} \mathbb{R}^{n}$ that satisfy the Theorem 2.7 (see also [3, 14]).

Example 4.2 Let $H$ be a $\sigma$-compact, locally compact connected group and for all $h \in H, \tau_{h}: \mathbb{R}^{n} \longrightarrow \mathbb{R}^{n}$ is given by $x \longmapsto \Delta_{H}(h) x$. Then $G=H \times_{\tau} \mathbb{R}^{n}$ is a locally compact group with $\delta=\Delta^{-1}$. So that $G$ is unimodular and so by Theorem 2.7(ii) the quasi regular representation $U$ is not square integrable. Also, one can check this directly. Indeed, it is clear that for every $\omega \neq 0$ in $\widehat{\mathbb{R}^{n}}$, the orbit of $\omega$ is the straight line that passes from $\omega$ and $(0,0, \ldots, 0)$ without the origin. Let $\alpha$ and $\beta$ be two nonzero points in $\widehat{\mathbb{R}^{n}}$ with different orbits. Then the cone obtained by $O_{\alpha}$ and $O_{\beta}$ in $\widehat{\mathbb{R}^{n}}$ is an invariant set. Hence, by [3] there are many reducible subrepresentations of $U$. In particular $U$ is not irreducible.

Example 4.3 It is easy to see that for every compact group $H$, the semidirect product $H \times{ }_{\tau} \mathbb{R}^{n}$ is unimodular. Hence, the quasi regular representation associated to $H \times{ }_{\tau}$ $\mathbb{R}^{n}$ is not square integrable.

Example 4.4 Let

$$
H=\left\{\left(\begin{array}{ccc}
x & 0 & y \\
0 & x^{-1} & 0 \\
0 & 0 & 1
\end{array}\right): x \neq 0, y \in \mathbb{R}\right\} .
$$

We observe that left Haar measure is $x^{-2} d x d y$ while right Haar measure is $|x|^{-1} d x d y$. So $H$ is not unimodular and $\Delta_{H} \not \equiv \delta^{-1}$. It is also clear that if $\left(\omega_{1}, \omega_{2}, \omega_{3}\right) \in \mathbb{R}^{3}$ with $\omega_{1} \omega_{2} \neq 0$, then the $\epsilon$-stabilizer $H_{\epsilon}^{\omega}$ is compact for $\epsilon>0$ 
sufficiently small. Hence, part (ii) of Theorem 4.1 shows that the quasi regular representation of $H \times{ }_{\tau} \mathbb{R}^{3}$ has an admissible vector.

Now we discuss an important example of semidirect product groups, i.e. the Heisenberg group, see [1, 3]. First we review $H(G)$, the Heisenberg group of an LCA group $G$, then we show that quasi regular representation on $H(G)$ is not square integrable. Note that the second component of this group is not $\mathbb{R}^{n}$, hence Theorem 4.1 would certainly fail this requirement.

Example 4.5 (The Heisenberg group) For every LCA group $G$, the Heisenberg group of $G$, is defined by $H(G)=G \times{ }_{\tau}(\widehat{G} \times T)$ in which for any $x \in G$ the automorphism $\tau_{x}$ on $\widehat{G} \times T$ is $\tau_{x}(\omega, z)=(\omega, z \omega(x))$. Also, the operations of $H(G)$ are defined as follows:

$$
\begin{aligned}
\left(x_{1}, \omega_{1}, z_{1}\right) \cdot\left(x_{2}, \omega_{2}, z_{2}\right) & =\left(x_{1} x_{2}, \omega_{1} \omega_{2}, z_{1} z_{2} \omega_{2}\left(x_{1}\right)\right), \\
(x, \omega, z)^{-1} & =\left(x^{-1}, \bar{\omega}, \bar{z} \omega(x)\right) .
\end{aligned}
$$

A straightforward calculation gives that $\delta \equiv 1$. Hence $H(G)$ is unimodular and $d x d \omega d z$ is its Haar measure. Now we are going to show that the quasi regular representation of $H(G)$ is not square integrable. To see this, we define the representation $\left(\pi, L^{2}(\widehat{G})\right)$ on $H(G)$ by

$$
(\pi(x, \omega, z) f)(\gamma)=z \gamma(x) \overline{\omega(x)} f(\gamma \bar{\omega}), \quad \text { for }(x, \omega, z) \in H(G), f \in L^{2}(\widehat{G}) .
$$

Lemma 4.6 The above representation $\left(\pi, L^{2}(\widehat{G})\right)$ is square integrable.

Proof Assume that $f, g \in L^{2}(\widehat{G})$ and $T_{\omega}$ is the translation operator on $L^{2}(\widehat{G})$. Then by Plancherel Theorem (4.25 of [6]) we have;

$$
\begin{aligned}
& \int_{H(G)}|\langle f, \pi(x, \omega, z) g\rangle|^{2} d z d x d \omega \\
& =\int_{\widehat{G}} \int_{G} \int_{T}\left|\int_{\widehat{G}} f(\gamma) \bar{z} \overline{\gamma(x)} \omega(x) \overline{g\left(\gamma \omega^{-1}\right)} d \gamma\right|^{2} d z d x d \omega \\
& =\int_{\widehat{G}} \int_{G}\left|\int_{\widehat{G}} f(\gamma) \overline{\gamma(x)}\left(T_{\omega^{-1}} \bar{g}\right)(\gamma) d \gamma\right|^{2} d x d \omega \\
& =\int_{\widehat{G}} \int_{G}\left|\left(f . T_{\omega^{-1}} \bar{g}\right)(x)\right|^{2} d x d \omega \\
& =\int_{\widehat{G}} \int_{\widehat{G}}\left|\left(f . T_{\omega^{-1}} \bar{g}\right)(\gamma)\right|^{2} d \gamma d \omega \\
& =\int_{\widehat{G}} \int_{\widehat{G}}|f(\gamma)|^{2}\left|\bar{g}\left(\gamma \omega^{-1}\right)\right|^{2} d \gamma d \omega=\|f\|_{2}^{2}\|g\|_{2}^{2} .
\end{aligned}
$$

It follows that every element in $L^{2}(\widehat{G})$ is cyclic and admissible. Hence $\left(\pi, L^{2}(\widehat{G})\right)$ is square integrable by Schur's Lemma (3.5 of [6]) [6]. 
If we consider $L^{2}(\widehat{G})$ as a closed subspace of $L^{2}(\widehat{G} \times T)$ via the embedding map $f \mapsto \widetilde{f}$ where $\widetilde{f}(\omega, z)=\bar{z} f(\omega)$ for all $f \in L^{2}(\widehat{G})$, then $\pi$ is a non-trivial square integrable subrepresentation of the quasi regular representation $U$ on $H(G)$ and so $U$ is not square integrable.

Acknowledgements We would like to thank S. Twareque Ali and H. Fuhr for making [7] available to us and suggestions for the final version of this paper. Thanks are also due to the referees for several valuable comments.

\section{References}

1. Ali, S.T., Antoine, J.P., Gazeau, J.P.: Coherent States, Wavelets and Their Generalizations. Springer, New York (2000)

2. Antoine, J., Murenzi, R., Vandergheynst, P., Ali, S.T.: Two-dimensional Wavelets and Wheir Relations. Cambridge University Press, Cambridge (2003)

3. Arefijamaal, A.A., Kamyabi-Gol, R.A.: A characterization of square integrable representations associated with CWT. J. Sci. I.R. Iran 18(2), 159-166 (2007)

4. Arefijamaal, A.A., Kamyabi-Gol, R.A.: On construction of coherent states associated with semidirect products. Int. J. Wavelets Multiresolut. Inf. Process. 6(5), 749-759 (2008)

5. Dubechies, I.: Ten Lectures on Wavelets. SIAM, Philadelphia (1992)

6. Folland, G.B.: A Course in Abstract Harmonic Analysis. CRC Press, Boca Raton (1995)

7. Fuhr, H.: Abstract Harmonic Analysis of Continuous Wavelet Transforms. Springer Lecture Notes in Mathematics, vol. 1863. Springer, Berlin (2005)

8. Fuhr, H.: Wavelet frames and admissibility in higher dimensions. J. Math. Phys. 37, 6353-6366 (1996)

9. Glimm, J.: Locally compact transformation groups. Trans. Am. Math. Soc. 101, 124-138 (1961)

10. Grossmann, A., Morlet, J., Paul, T.: Transforms associated to square integrable group representation I. General results. J. Math. Phys. 26, 2473-2479 (1985)

11. Hewitt, E., Ross, K.A.: Abstract Harmonic Analysis, vols. 1, 2. Springer, Berlin (1970)

12. Kamyabi-Gol, R.A., Tavallaei, N.: Wavelet transforms via generalized quasi-regular representations. Appl. Comput. Harmon. Anal. (to appear)

13. Koornwinder, T.H.: Wavelets: An Elementary Treatment of Theory and Applications. World Scientific, Singapore (1993)

14. Laugesen, R.S., Weaver, N., Wiess, G.L., Willson, E.N.: A characterization of the higher dimensional groups associated with continuous wavelets. J. Geom. Anal. 12, 89-102 (2002) 